\title{
The Amigo Service Architecture for the Open Networked Home Environment
}

\author{
Nikolaos Georgantas $^{1, \mathrm{a}}$, Sonia Ben Mokhtar ${ }^{1, \mathrm{a}}$, Yérom-David Bromberg ${ }^{1, \mathrm{a}}$, Valérie Issarny ${ }^{1, \mathrm{a}}$, Jarmo \\ Kalaoja $^{2, b}$, Julia Kantarovitch ${ }^{2, b}$, Anne Gérodolle ${ }^{3, \mathrm{c}}$, Ron Mevissen ${ }^{4, \mathrm{~d}}$ \\ ${ }^{1}$ INRIA, ${ }^{2}$ VTT, ${ }^{3}$ France Télécom, ${ }^{4}$ Microsoft \\ ${ }^{a}\left\{\right.$ first.lastname@inria.fr\}, ${ }^{b}$ \{first.lastname@vtt.fi\}, ${ }^{c}$ anne.gerodolle@francetelecom.com, ${ }^{d}\{$ ronme@microsoft.com\}
}

\begin{abstract}
The Amigo project aims to develop a networked home system enabling the ambient intelligence / pervasive computing vision by effectively integrating devices and their hosted services in today's home. The Amigo system architecture poses limited technology-specific restrictions, supporting interoperability among heterogeneous services.
\end{abstract}

\section{Introduction}

The open networked home environment shall allow the seamless integration of the various devices and applications coming from today's, mostly disjoint, four home application domains: personal computing (PC), based on the home computers and Internet connection; mobile computing, manifested by the increasing use of home Wi-Fi and Bluetooth networks connecting laptops, PDAs and tiny personal devices; consumer electronics (CE), targeting multimedia in the home; and home automation, adding intelligence to household appliances. Service-orientation [3] appears as the appropriate architectural paradigm for the networked home system. Therein, networked devices and hosted applications are abstracted as services, which may dynamically be retrieved and composed, thanks to service discovery and coordination protocols.

This paper outlines the Amigo service-oriented system architecture for the open networked home, which has been designed as part of the Amigo ${ }^{1}$ European project on the development of a networked home system enabling the ambient intelligence / pervasive computing vision. Key property that we are targeting within Amigo is interoperability, since the networked home integrates devices coming from different manufacturers that use different communication standards and different hardware and software platforms. The novelty of the Amigo approach lies in imposing no specific middleware technology, as most existing service architectures do: it allows heterogeneous technologies to be integrated, establishing interoperability at a higher, semantic, level. Further properties provided by the Amigo system are context-awareness, quality of service (QoS)

${ }^{1}$ http://www.hitech-projects.com/euprojects/amigo/ and security \& privacy, as these decisively affect user's experience of the networked home. In the next section, we introduce the overall Amigo reference service architecture; we conclude in Section 3.

\section{Amigo Reference Service Architecture}

In the elaboration of the Amigo system architecture, we do not intend to develop yet another service infrastructure imposing a homogeneous middleware layer on all home devices. We elaborate an abstract reference service architecture for the Amigo system, which can represent and integrate existing individual service infrastructures (e.g., Web Services ${ }^{2}$, $\mathrm{UPnP}^{3}$, etc.) relevant to the four home application domains by abstracting their fundamental features. To specify the Amigo reference architecture, we use as basis a typical service-oriented architecture [3]. The required properties discussed in Section 1 lead us to introduce additional building blocks into the typical architecture. Principally, we assume that all three layers (application-middleware-platform) of the architecture may be heterogeneous and based on diverse technologies; therefore, we incorporate appropriate interoperability mechanisms. The resulting Amigo reference architecture is depicted in Figure 1, where the advanced added elements are printed in boldface [2].

The interoperability mechanisms are based on semantics. Semantics of an entity encapsulate the meaning of this entity by reference to an established vocabulary of terms (ontology) representing a specific area of knowledge. In this way, semantics of entities become machine-interpretable, enabling machine reasoning on them. Such concepts come from the knowledge representation field and have been applied and further evolved in the Semantic $\mathrm{Web}^{4}$ domain. We have employed semantic concepts to model Amigo services. Our semantic service modeling builds upon the software architecture notions of components and connectors [1]. Figure 2 depicts the mapping of the

\footnotetext{
${ }^{2}$ http://www.w3.org/2002/ws/

${ }^{3} \mathrm{http}: / /$ www.upnp.org

${ }^{4}$ http://www.w3.org/2001/sw/
} 
Amigo service modeling on the Amigo reference architecture. Typically, components are mapped on the application layer, and connectors are mapped on the middleware layer, to which we may attach the platform layer in order to address the complete interaction mechanism offered to applications. What distinguishes our mapping from the typical case is the semantic modeling and the resulting interoperability feature. Based on this modeling, we have introduced generic conformance relations at both component/application and connector/middleware levels, which enable reasoning on service composability. We have further devised associated interoperability methods at both component/application and connector/middleware levels, which allow composing partially conforming services. Conformance relations together with interoperability methods establish semantics-based service interoperability [1].

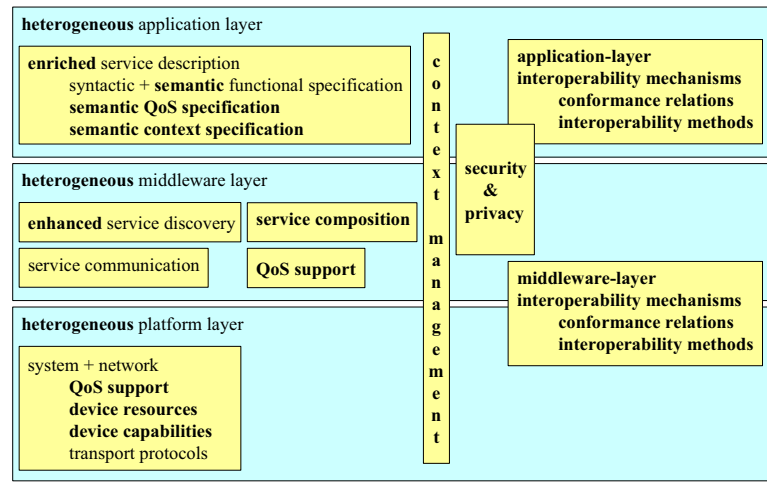

Figure 1. Amigo reference service architecture

Based on the Amigo reference service architecture and the encompassed semantics-based interoperability, we further define a device capable of being integrated into the Amigo system as any device that: (i) implements (a subset of) the reference architecture employing specific technologies; and (ii) may implement some interoperability methods. This definition is very generic and allows very diverse technologies to be integrated into the Amigo system. The incorporation of interoperability methods by a device within Amigo will depend on a number of factors, such as the feasibility to enhance legacy platforms, the computational resources of the device, and certainly the necessity of such methods. Two devices capable of being integrated into the Amigo system will be interoperable if: (i) they conform semantically in terms of functional and non-functional properties; and (ii) they implement complementary interoperability methods. The degree to which the first condition is true, determines the degree to which the second condition is needed. Moreover, the complementarity of interoperability methods allows deploying such methods on one or both peer devices, for example depending on their resource capacity, or even on a third, intermediate, node (gateway).

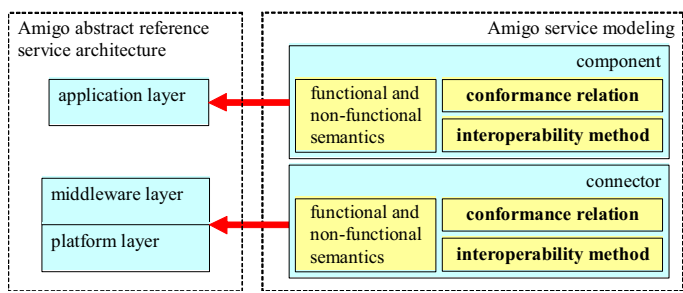

Figure 2. Amigo service modeling

Applying the above fundamental principles, the Amigo reference architecture supports a number of features [2], such as: (i) (in the application layer) enriched service description specifying both functional and non-functional (context \& QoS) properties of services; (ii) (in the middleware layer) service communication, enhanced service discovery reflecting the enriched service description, service composition, $Q o S \&$ context support, security \& privacy; (iii) (in the platform layer) QoS support, device resource- \& capability-awareness.

\section{Conclusion}

We have outlined the Amigo reference architecture integrating heterogeneous devices and hosted services in the networked home environment by establishing interoperability at semantic level. Our next objectives in the Amigo project concern the detailed specification and prototype implementation of the Amigo system architecture. In this direction, specific application-layer and middleware-layer interoperability methods are being developed, as presented in $[4,5,6]$.

\section{References}

[1] N. Georgantas, S. Ben Mokhtar, F. Tartanoglu, V. Issarny. Semantics-Aware Services for the Mobile Computing Environment. In Architecting Dependable Systems III, LNCS, Springer-Verlag, 2005 (to appear).

[2] Amigo Consortium. Deliverable D2.1: Specification of the Amigo Abstract Middleware Architecture. April 2005.

[3] M. P. Papazoglou, D. Georgakopoulos (eds.). Serviceoriented computing. Special section in Communications of the ACM, Volume 46, Issue 10, October 2003.

[4] S. Ben Mokhtar, J. Liu, N. Georgantas, V. Issarny. QoSaware Dynamic Service Composition in Ambient Intelligence Environments. Short paper. In Proceedings of ASE 2005, November 2005 (to appear).

[5] Y.-D. Bromberg, V. Issarny. INDISS: Interoperable Discovery System for Networked Services. In Proceedings of Middleware 2005, November 2005 (to appear).

[6] Amigo Consortium. Deliverable D3.1: Detailed Design of the Amigo Middleware Core. September 2005. 